\title{
Context and Short Term User Intention Aware Hybrid Session Based Recommendation System
}

\author{
Ramazan Esmeli \\ School of Computing \\ University of Portsmouth \\ Portsmouth, United Kingdom \\ ramazan.esmeli@port.ac.uk
}

\author{
Mohamed Bader-El-Den \\ School of Computing \\ University of Portsmouth \\ Portsmouth, United Kingdom \\ mohamed.bader@port.ac.uk
}

\author{
Alaa Mohasseb \\ School of Computing \\ University of Portsmouth \\ Portsmouth, United Kingdom \\ alaa.mohasseb@port.ac.uk
}

\begin{abstract}
Information overloading in e-commerce hinders the consumers' ability to make the right decisions. Customers visiting e-commerce websites can have specific goals in an individual session. However, using sessions that are based on the last item viewed or purchased is not enough to exploit the sessions specific intention or predict users' next actions in the sessions. In this paper, we proposed context and short term user intention aware (CSUI) framework which is based on item similarity collaborative filtering and Association Rule Session-based recommendation systems, the proposed model combines context factor of users' session and users' short term intentions. The developed model has been evaluated on two real-world datasets. Experimental results showed that using session context and users' short term intentions during the recommendation process could help in improving the accuracy of the next item prediction.
\end{abstract}

Index Terms - Context awareness, short term user intention, recommendation systems, session-based recommendation

\section{INTRODUCTION}

Information overloading in e-commerce hinders the consumers' ability to make the right decisions. In the past two decades, Recommendation Systems (RS) have been successful in supporting both customers in exploring relevant items, and marketers in improving their marketing strategies. In general, the success of RS is valued by its ability of matching consumer and items. However, in many real online retails scenarios, there is very little information available about each consumer, e.g. many consumers browse e-commerce websites without logging in. According to a dataset realised by DIGINETICA ${ }^{1}$, it is found that $62.3 \%$ of the users are not logged in to the system [1]. In addition, recommendations have to depend on ongoing user interactions [2], which means that each session for unregistered users in e-commerce domain is considered as a new starting session. Furthermore, in traditional collaborative filtering RS, Matrix Factorisation (MF) [3] methods have obtained promising results when users have a long history. However, MF methods suffer since non-logged users do not have hidden features to represent them.

Furthermore, Session-based Recommendation Systems (SRS) have become an essential component of e-commerce platforms, since its ability to recommend items to anonymous users who do not have long term histories. SRS could also be used to make suggestions for the next possible users' actions

\footnotetext{
${ }^{1}$ http://diginetica.com/
}

(e.g. view and click) based on sequential user behaviour in a session [4]. Moreover, different methods have been developed for SRS including item similarity based collaborative filtering [5] which gives recommendations based on item similarity, Markov Chain (MC) [6] which uses sequential relations between the user actions to make predictions and Factorised Markov Chain (FPMC) [7], which is a model of the combination of MC and MF methods.

Recently, Recurrent Neural Network (RNN) based SRS have emerged. Authors in [8] have proposed a deep neural network based RS which is also considered as a baseline model for the deep learning based SRS, later in [4] the model has been extended, and new attributes have been added such as item and user related attributes.

RNN models consider the sequential awareness in a session such as the connections of sequentially clicked items [1], and showed better accuracy results over the base item similarity CF model. However, experiment results showed that newly developed versions of the item based CF models could achieve similar or even better accuracy results than deep learning based models [9], [10]. Also, item-based CF models are still commonly used in practise for SRS [8], [9].

Furthermore, traditional sequential pattern models (e.g. Item-Item KNN, Associated Rules (AR)) take into account last clicked item as user intention in the session rather than considering previously interacted items in an ongoing session which might cause losing valuable information about user intentions. For example, previously viewed items by a user could be less interesting than the last clicked item but might still have more value and could help in determining user intentions. Moreover, in an ongoing session, if the user mostly visited a specific type of item categories, the user is more likely interested in this category type of items. Most traditional SRS approaches and deep learning based approaches do not take into account this kind of valuable information to identify user intentions.

In this paper, we proposed a novel context and short term user intention aware (CSUI) framework which is based on item similarity collaborative filtering and Association Rule Session-based recommendation systems, and the proposed model combines context factor of users' session and users' short term intentions. Also, an analysis has been provided 
for the effect of integrating short term user intention and context awareness for an on-going session to take into account user current intention during the recommendation process. The contributions of this paper are as followed:

1) A model to investigate sequence awareness of item similarity CF and Association Rules (AR) based models for the SRS.

2) A model to investigate short term user intention on item similarity $\mathrm{CF}$ and Association Rules (AR) based SRS

3) A new post context filtering approach for the item similarity CF and Association Rules (AR) based SRS.

4) Quantitative analysis of two real e-commerce datasets to show the effectiveness of the proposed method over the recommendation methods.

We used two real-world e-commerce datasets. Experimental results imply that the proposed model demonstrates a good level of accuracy in next item predictions compared to baseline item-item KNN and AR models.

The rest of the paper is organised as follows. Section 2, provides a review of related works. Section 3, describes the proposed framework. The experiments setup and results are presented in Section 4. Finally, Section 5 concludes the paper and outlines directions for future work.

\section{RELATED WORKS}

In this section, we review previous work on the different type of session-based recommendation systems models.

\section{A. Item Based Collaborative Filtering}

Item-based collaborative filtering (CF) [11] is the most used type of recommendation systems in different e-commerce domains to recommend items for unregistered users. [8], [9]. Also, the item-based CF method is a strong baseline on the comparison of deep learning approaches for SRS performance [8], [4]. Item similarity based CF method is used in the Amazon [5] website to have a real-time recommendation and scaled model. Authors in [12] proposed a session based item similarity model ( $\mathrm{S}-\mathrm{KNN})$ the proposed model measures most similar session to the current session, and the items are ranked based on their weights which are calculated in the most similar session. In addition, a Temporal KNN (T-KNN) model was proposed in [9], authors have introduced a temporal awareness to the item similarity $\mathrm{CF}$ model by changing the last item in the session for the next recommendations. In our case, we considered the whole session as a context for the user and based on the all viewed item and last viewed item we created a recommendation list.

\section{B. Association Rule-Based Recommendation}

Association rule generates rules for discovering frequently appeared items in the individual transactions by data analysing techniques. Transactions in the SBRS could be considered as the subsequent items in a session [13]. Moreover, association rule has been used in the recommendation systems for alleviating the cold start problems[14]. Authors in [15] used AR for SRS to compare the performance to other models, including deep learning based SRS. Furthermore, authors in [16] proposed a method for anonymous users to recommend food with association rule based recommendation. However, in both works mentioned above, AR models were used to give the next item recommendation based on the currently viewed item.

\section{Sequence Aware Recommendation}

Sequence aware Recommendation Systems (RS) have become one of the most used recommendation systems in the e-commerce domain. MC models applied in many works for next item prediction [6], and these models also have sequence awareness since the transition matrix is used to predict the next item. However, the sequence length of MC models for each session is fixed in other means, and MC models can consider an only certain number of previous items for each session. Authors in [8] adopted recurrent neural networks for recommendation systems. In their method, a session consists of a sequence of items and next product recommendations affected by all previous items in a session. In addition, the proposed model do not have category awareness and the level of given importance to the user last clicked item is not certain.

\section{Time Aware Recommender Systems}

Time-weighted collaborative filtering was proposed by [17]. The main aim of their method is to use the user interest drift over time; in other words, recent user ratings on items' should be more representative for user preferences than old rated items. Therefore, they designed an algorithm that gives more weight to recently rated items. Our work has a similar approach to this research. However, we will not use this approach in the long term user history since in our dataset we have short term user interactions. Moreover, time aware RS applied to different domains including user point of interest (POI) [18], Movie [3], e-commerce[19] and showed better performance comparing to user interest drift unaware models.

\section{E. Context Aware Recommendation}

Context-aware RS takes into consideration the context data in the moment recommendation asked [20]. Users are acting differently when the context changed [20] and browsed items in a session can be assumed as context [2], [21]. Authors in [21] proposed a method to adopt long term and short term user history data. In their work, they applied a contextualisation strategy by applying a refined method to recent user actions. In their contextualisation strategy, they have different approaches including feature matching. All these approaches could be considered as contextual-post filtering. In our work, we use a similar feature matching method to refine the recommendations. However, our approach is different in terms of how the contextual-post filtering is applied.

\section{Context And Short Term Intention Aware RS}

In this section, we describe the context and short term user intention aware (CSUI) framework. The motivation of this framework is to have more personalised recommendations, in 


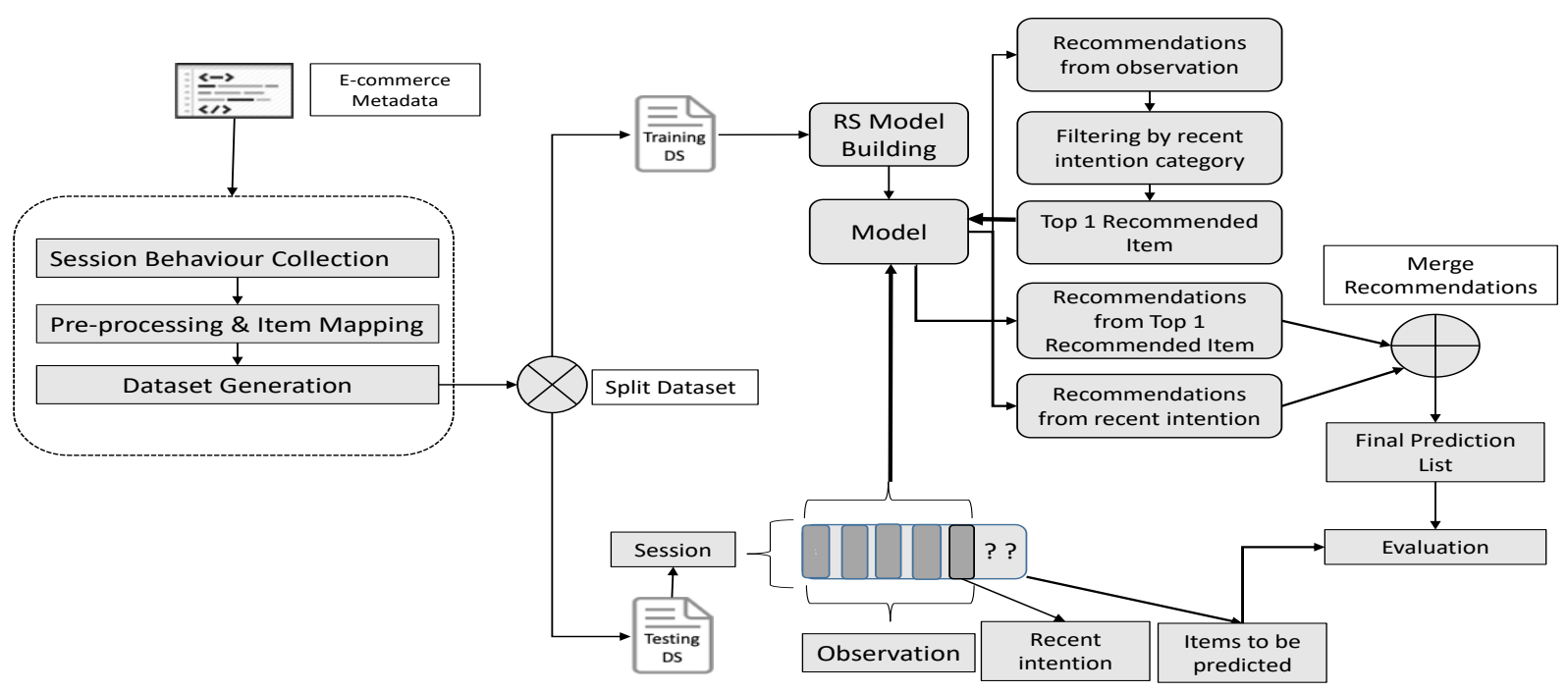

Fig. 1. Context and Short Term User Intention Aware (CSUI) RS framework

which users are anonymous, and the system does not know the users' product interaction history besides the ongoing session. This framework helps improving the accuracy of next item prediction in the ongoing session by producing more attention to users' short term goal and the users' contexts in the session. In this framework, short term means the last interacted item; context means all the interacted items and their features in the session. The framework consists of three stages;

\section{A. Stage 1: Dataset Pre-Processing}

In this stage, the main aim is to prepare the data for RS algorithms and creating a train, test datasets for the validation.

\section{B. Stage 2: Model Training}

This stage consists of training of the models on training dataset; in this paper, we used two different models, which are item similarity CF and association rules-based model.

\section{Stage 3: Recommendation and Validation}

The contribution of our work is more focused on this stage of the framework. In this phase, we increase the personalisation of the recommendations by applying short term intention and context awareness.

As seen in Figure 1, framework learns users' intention from sequentially interacted items. For example, if a user interacted only with one item in the session, this item will be used to get suggestions, and the suggested items are filtered based on this item's feature. In case the user interacted with more than one item in the session, a final recommendation is created using all interacted items, however, the items in the already interacted list have different contributions to the last recommendation list, in which items closer to last interacted item have more contribution since they are closer to uses' current intention. After having a final recommendation list from the interacted items, feature filtering applied to the final list to have more related recommendations to users' current purpose and item which has the highest confidence level in the final list is selected to retrieve recommendations (contextaware recommendation list) from the model. In addition, filtering is not applied to the recommendation list (short term recommendation list) gathered base on the last interacted item. Final ranked recommendation list is created from the combination of context and short term aware recommendations.

1) Interacted Items Weights Calculation: Another advantage of this framework is the use of a weighting model for the context awareness in order to increase personalisation in the recommendations.

The main aim of using weighting model is that instead of using the recommendations based only on the last interacted item, which takes into account only the previously viewed items to have a context awareness of the whole session for the user since user's final target may have affected from entire session. We have assumed that the previously interacted items may have slightly different effects on the ultimate users' intention based on their positions. Thus, we designed a weighting model to adjust the previously viewed items' augmentations to the next product recommendations. The weights are calculated as follows: Let say the index of last interacted item in the session is $P$ and previously interacted items has the index $p$ $\in\{P-1, P-2, . .0)\}$.

$$
W(P, p)= \begin{cases}\frac{1}{2 P}, & \text { if } p=0 \\ \frac{p}{P}, & \text { otherwise }\end{cases}
$$

As seen from weight calculation, we assumed that the currently clicked item has more impact on producing next item predictions. Moreover, the weighting method eliminates recommended items, which suggested as a result of the interaction 


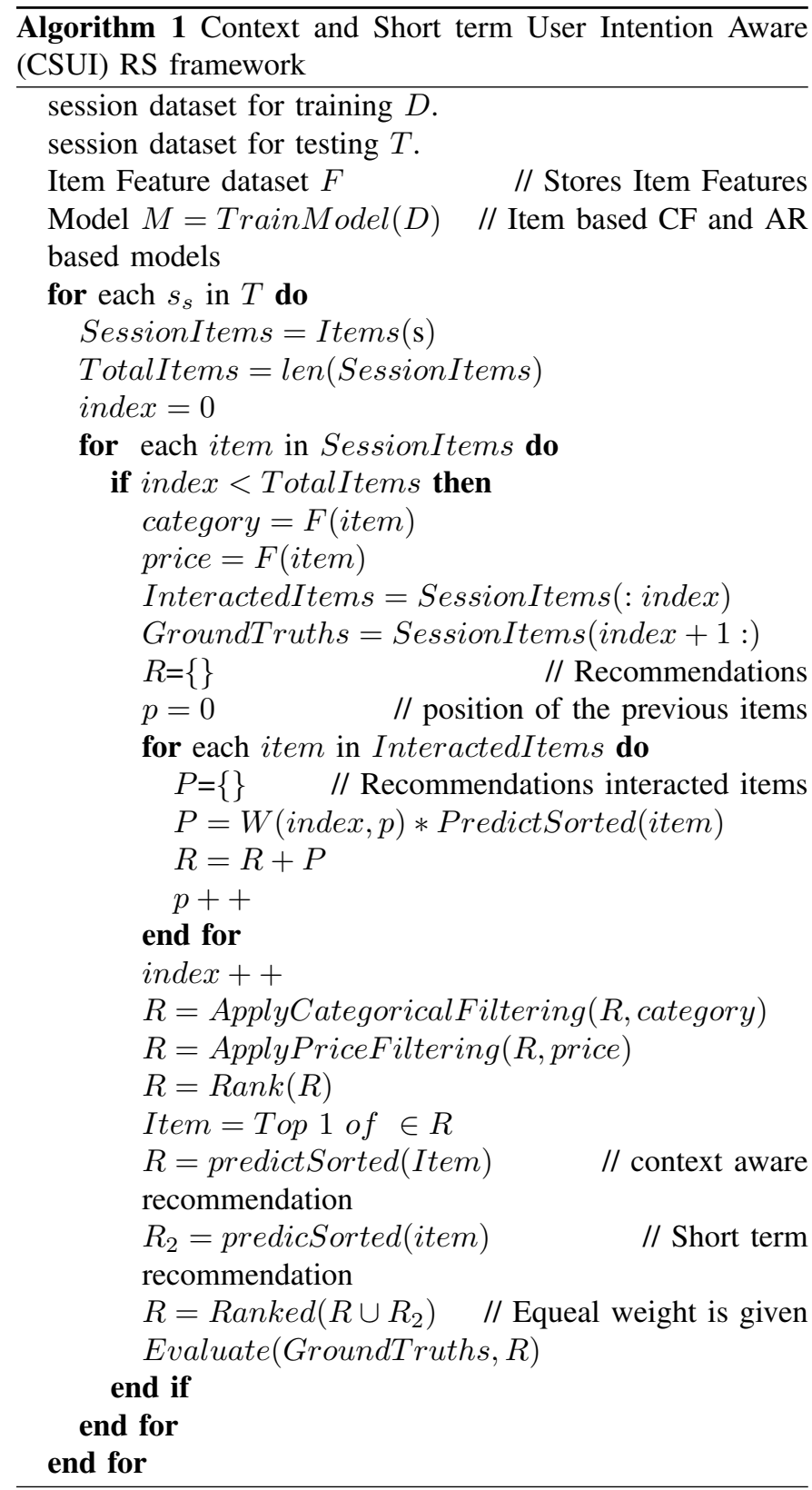

of the current item, if they have less confidence level than recommended items as a consequence of the previous interacted items.

The score sets(similarity or the confidence values of the recommended items) of the recommendations from each items in different positions are $S \in\left\{S_{P}, S_{P-1} . ., S_{0}\right\}$, so the scores of the final recommendation list $R_{S}$ is calculated in Eq 1

$$
\operatorname{score}\left(R_{S}\right)=\sum_{p=0}^{P} w_{p} S_{p}
$$

2) Evaluation: The Datasets were divided as test and train. To measure the performance of the CSUI framework, recall@N, precision@N are used. This evaluation aims to see how the proposed framework performs on predicting the next items that user can view in the sessions as shown in Figure 1.

\section{EXPERIMENTS AND RESULTS}

\section{A. Datasets}

Two real-world e-commerce datasets were used to test the effectiveness of CSUI framework: 1) Dataset A is yoochose dataset which is used in ACM recsys competition in 2015 2 , 2) Dataset $\mathbf{B}$ is an e-commerce dataset which stores two weeks users' session data, this dataset was collected by a personalisation company in the UK (due to data privacy, we will refer to this dataset as CoDS). Both datasets consist of item features such as category and price which help filtering relevant items. In the pre-processing stage, sessions which contain less than two interacted items are excluded from the datasets. After the split of the testing and training dataset, the items appeared in the test dataset but not in the train dataset are filtered out. As the last step of pre-processing, the sessions which are consisted of less than two items are filtered out from both the training and testing datasets in order to validate our approach. Dataset A; the training dataset has 31579006 interactions, 7953885 unique sessions, 37483 unique items. In addition, there are 58233 interactions, 12372 unique sessions and 6359 unique items in the testing dataset.

Dataset B; the training dataset has 3837166 total interaction, 579040 unique sessions, 29935 unique items. The testing dataset consists of 680661 interactions, 65845 unique sessions and 27647 unique items. The items frequency in the sessions of both yoochose and second dataset are mostly two items which means we have minimal information about these sessions to create connections with other sessions.

Since we use categories of the items to filter the recommendations, category statistics of the two datasets are examined. The highest frequency for yoochose dataset in the product category is $\mathbf{S}$ and trainers for the second dataset as shown in Figure 2.

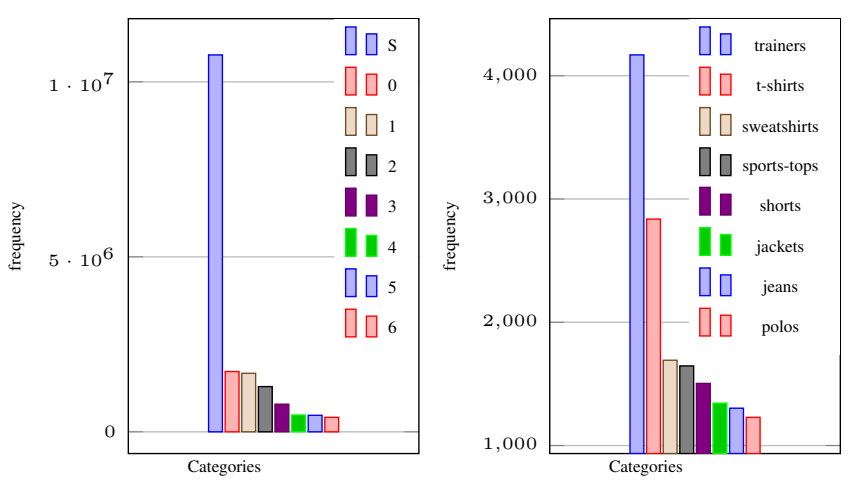

Fig. 2. Top 8 product categories appeared in the yoochose and the second dataset respectively

\section{B. Evaluation metrics}

To assess the performance of our approach (CSUI), the proposed framework is evaluated using precision, recall metrics.

\footnotetext{
${ }^{2} \mathrm{https} / / /$ recsys.acm.org/recsys15/challenge/
} 
TABLE I

PERFORMANCE COMPARISON OF THE PROPOSED FRAMEWORK ON THE ACCURACY OF LAST 1 ITEM PREDICTION IN THE SESSIONS (F: BASE FILTERED, CIA: Context AND INTENTION AwARE, CIAF: Filtered CIA)

\begin{tabular}{lllllllll}
\hline Dataset & \multicolumn{2}{c}{ Yoochose } & \multicolumn{2}{c}{ CoDS } & \multicolumn{2}{c}{ Yoochose } & \multicolumn{2}{c}{ CoDS } \\
Metric & R $@ 20$ & P@ 20 & R@20 & P@ 20 & R@ 30 & P@ 30 & R@ 30 & P@ 30 \\
\hline KNN & 0.4700 & 0.1700 & 0.4688 & 0.1685 & 0.5166 & 0.1253 & 0.5033 & 0.1203 \\
KNN-F & 0.4700 & 0.1700 & 0.4677 & 0.1685 & 0.5166 & 0.1253 & 0.5022 & 0.1206 \\
KNN-CIA & $\mathbf{0 . 6 6 7 7}$ & $\mathbf{0 . 2 4 3 5}$ & 0.4711 & 0.1690 & $\mathbf{0 . 7 0 4 4}$ & $\mathbf{0 . 1 7 1 6}$ & 0.5055 & 0.1210 \\
KNN-CIAF & 0.6377 & 0.2355 & $\mathbf{0 . 5 0 5 5}$ & $\mathbf{0 . 1 8 3 0}$ & 0.6844 & 0.1683 & $\mathbf{0 . 5 5 8 8}$ & $\mathbf{0 . 1 3 4 3}$ \\
\hline AR & 0.4711 & 0.1690 & 0.4900 & 0.1742 & 0.5322 & 0.1273 & 0.5277 & 0.1263 \\
AR-F & 0.4711 & 0.1690 & 0.4688 & 0.1672 & 0.5322 & 0.1273 & 0.5055 & 0.1213 \\
AR-CIA & 0.4722 & 0.1695 & 0.4866 & 0.1742 & 0.5288 & 0.1263 & 0.5255 & 0.1258 \\
AR-CIAF & $\mathbf{0 . 6 2 5 5}$ & $\mathbf{0 . 2 2 8 0}$ & $\mathbf{0 . 5 3 2 2}$ & $\mathbf{0 . 1 9 1 2}$ & $\mathbf{0 . 6 7 7 7}$ & $\mathbf{0 . 1 6 4 3}$ & $\mathbf{0 . 5 9 3 3}$ & $\mathbf{0 . 1 4 2 8}$ \\
\hline
\end{tabular}

In RS domain, recall@ $N$ is the ratio between the length of correct predicted items within top $N$ recommendations and the length of test items (Ground truth) and precision $@ N$ is the ratio between the length of correct predicted items within top $N$ recommendations and the length of total recommended items. They are computed as follows:

$$
\begin{gathered}
\text { Recall }=\frac{\mid \text { relevant recommendations } \mid}{\mid \text { relevant } \text { items } \mid} \\
\text { Precision }=\frac{\mid \text { relevant recommendations } \mid}{\mid \text { total recommendations } \mid}
\end{gathered}
$$

After having final ranked recommended items, we have evaluated the proposed models with above mentioned metrics for $N=20$ and $N=30$.

\section{Experiments}

We conducted four different experiments for each dataset to assess the performance of our approach CSUI. The task in this experiments is to predict the last 1 product in the user's sequence that the user viewed in the sessions. To measure this, we used the trained models to give recommendations based on the given interacted items, then we applied the methods described below, finally, we filtered top@n, $\mathbf{n} \in\{\mathbf{2 0 , 3 0}\}$, recommendation and evaluated with recall,precision metrics;

1) Baseline: In the first experiment, we evaluate the base item similarity $\mathrm{CF}$ and $\mathrm{AR}$. In this case, next item predictions depend only on the last interacted item. Thus, the model does not have sequence awareness and context awareness.

2) Base Filtered: In the second experiment, we evaluated feature matching over the base model in which feature filtering is applied over the recommendations based on last interacted item of the session. This model also does not have sequence awareness since the next items depend on the last interacted item in the session.

3) Context and Users' short term intention Aware: In the third experiment, we evaluated the context and short term intention aware model. As explained in Section 3, context means here the effect of users' previous interacted items in the session on determining the next viewed items. The aim of this experiment is to examine the effect of the context of previous interactions and short term user intention awareness on the overall performance.
4) Context and Users' short term intention Aware Filtered: In the last experiment, we evaluated the performance of the proposed framework when feature filtering over recommendations from previous interactions is applied to analyse the effectiveness of the feature filtering.

We tested the models with 1000 sessions. To choose test sessions, we iterated selection process ten times over the test dataset and in each iteration 100 sessions are randomly selected, overlapping of new selected 100 sessions with previously selected sessions are prevented to minimize the risk that the obtained results are specific to the single test split as shown in Figure 3. Finally, the performance measurements of all splits are averaged.
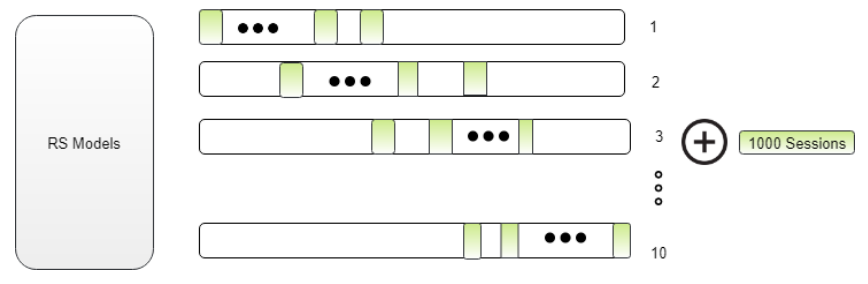

Fig. 3. Selecting test sessions to validate CSUI framework

\section{Results}

The results of the experiments show that adding context and short term intention awareness to item similarity and rulebased association recommendations performs well comparing to baseline models. Especially, the proposed framework boosted the performance of item similarity model approximately $40 \%$ over the baseline model. Applying feature matching filtering to base models did not make any improvement on both models. However, after integrating context and short term intention awareness to the models filtering approach showed the superiority in association rule model and improved the performance of about $31 \%$ over the base model.

\section{COnClusion And Future Works}

In this paper, we proposed the context and short term user intention aware (CSUI) framework. In addition, we investigated context and users' short term intention awareness in item similarity and associated rule-based recommendations. Both models are described as users' sequence unaware in several 
works [8], [4]. Experiment results show that our solution led to a good performance over the baseline models when applied on two different datasets.

As future works, the model effectiveness could be examined on other RS models, including MC. Also, the framework could be tested with other session-based datasets. Additionally, different approaches could be utilised to calculate the weights of the previously seen items in the sessions. Moreover, combining the context of the session and short term user intention could be performed using different weights. Another future direction can be using machine learning classification methods, including text classification [22] which can help to predict feature specifications of products from the user search query that the user might click or browse. However, in this type classification approaches class imbalance is the main challenge [23], [24] and to process data in real-time, tools such as Apache Spark [25], [26], need to be considered, in which recommendation process need to be done within a few seconds.

\section{ACKNOWLEDGE}

RE gratefully acknowledges the financial support of the Turkish Government (law 1416, YLSY), Ministry of Education for his $\mathrm{PhD}$ scholarship.

\section{REFERENCES}

[1] C. Wu and M. Yan, "Session-aware information embedding for ecommerce product recommendation," in Proceedings of the 2017 ACM on conference on information and knowledge management, pp. 23792382, ACM, 2017.

[2] M. Quadrana, P. Cremonesi, and D. Jannach, "Sequence-aware recommender systems," arXiv preprint arXiv:1802.08452, 2018.

[3] Y. Koren, "Collaborative filtering with temporal dynamics," in Proceedings of the 15th ACM SIGKDD international conference on Knowledge discovery and data mining, pp. 447-456, ACM, 2009.

[4] B. Hidasi, M. Quadrana, A. Karatzoglou, and D. Tikk, "Parallel recurrent neural network architectures for feature-rich session-based recommendations," in Proceedings of the 10th ACM Conference on Recommender Systems, pp. 241-248, ACM, 2016.

[5] G. Linden, B. Smith, and J. York, "Amazon. com recommendations: Item-to-item collaborative filtering," IEEE Internet computing, no. 1, pp. 76-80, 2003.

[6] W. Gu, S. Dong, and Z. Zeng, "Increasing recommended effectiveness with markov chains and purchase intervals," Neural Computing and Applications, vol. 25, no. 5, pp. 1153-1162, 2014.

[7] S. Rendle, C. Freudenthaler, and L. Schmidt-Thieme, "Factorizing personalized markov chains for next-basket recommendation," in Proceedings of the 19th international conference on World wide web, pp. 811-820, ACM, 2010.

[8] B. Hidasi, A. Karatzoglou, L. Baltrunas, and D. Tikk, "Sessionbased recommendations with recurrent neural networks," arXiv preprint arXiv:1511.06939, 2015.

[9] M. Ludewig and D. Jannach, "Evaluation of session-based recommendation algorithms," User Modeling and User-Adapted Interaction, vol. 28 , no. 4-5, pp. 331-390, 2018

[10] D. Jannach and M. Ludewig, "When recurrent neural networks meet the neighborhood for session-based recommendation," in Proceedings of the Eleventh ACM Conference on Recommender Systems, pp. 306310, ACM, 2017.

[11] B. M. Sarwar, G. Karypis, J. A. Konstan, J. Riedl, et al., "Itembased collaborative filtering recommendation algorithms.," $W w w$, vol. 1, pp. 285-295, 2001.

[12] S. E. Park, S. Lee, and S.-g. Lee, "Session-based collaborative filtering for predicting the next song," in Computers, Networks, Systems and Industrial Engineering (CNSI), 2011 First ACIS/JNU International Conference on, pp. 353-358, IEEE, 2011.
[13] R. Agrawal, T. Imieliński, and A. Swami, "Mining association rules between sets of items in large databases," in Acm sigmod record, vol. 22, pp. 207-216, ACM, 1993.

[14] G. Shaw, Y. Xu, and S. Geva, "Using association rules to solve the coldstart problem in recommender systems," in Pacific-Asia Conference on Knowledge Discovery and Data Mining, pp. 340-347, Springer, 2010.

[15] I. Kamehkhosh, D. Jannach, and M. Ludewig, "A comparison of frequent pattern techniques and a deep learning method for sessionbased recommendation.," in RecTemp@ RecSys, pp. 50-56, 2017.

[16] T. Osadchiy, I. Poliakov, P. Olivier, M. Rowland, and E. Foster, "Recommender system based on pairwise association rules," Expert Systems with Applications, vol. 115, pp. 535-542, 2019.

[17] Y. Ding and X. Li, "Time weight collaborative filtering," in Proceedings of the 14th ACM international conference on Information and knowledge management, pp. 485-492, ACM, 2005.

[18] Q. Yuan, G. Cong, Z. Ma, A. Sun, and N. M. Thalmann, "Timeaware point-of-interest recommendation," in Proceedings of the 36th international ACM SIGIR conference on Research and development in information retrieval, pp. 363-372, ACM, 2013.

[19] V. Bogina, T. Kuflik, and O. Mokryn, "Learning item temporal dynamics for predicting buying sessions," in Proceedings of the 21 st International Conference on Intelligent User Interfaces, pp. 251-255, ACM, 2016.

[20] G. Adomavicius and A. Tuzhilin, "Context-aware recommender systems," in Recommender systems handbook, pp. 217-253, Springer, 2011.

[21] D. Jannach, L. Lerche, and M. Jugovac, "Adaptation and evaluation of recommendations for short-term shopping goals," in Proceedings of the 9th ACM Conference on Recommender Systems, pp. 211-218, ACM, 2015.

[22] A. Mohasseb, M. Bader-El-Den, H. Liu, and M. Cocea, "Domain specific syntax based approach for text classification in machine learning context," in 2017 International Conference on Machine Learning and Cybernetics (ICMLC), vol. 2, pp. 658-663, IEEE, 2017.

[23] M. Bader-El-Den, E. Teitei, and T. Perry, "Biased random forest for dealing with the class imbalance problem," IEEE transactions on neural networks and learning systems, 2018.

[24] M. Bader-El-Den, "Self-adaptive heterogeneous random forest," in 2014 IEEE/ACS 11th International Conference on Computer Systems and Applications (AICCSA), pp. 640-646, IEEE, 2014.

[25] C. Diedhiou, B. Carpenter, and R. Esmeli, "Comparison of platforms for recommender algorithm on large datasets," in 7th Imperial College Computing Student Workshop, pp. 4-1, Schloss Dagstuhl-Leibniz Center for Informatics, 2019.

[26] C. Diedhiou, B. Carpenter, A. Shafi, S. Sarkar, R. Esmeli, and R. Gadsdon, "Performance comparison of a parallel recommender algorithm across three hadoop-based frameworks," in 2018 30th International Symposium on Computer Architecture and High Performance Computing (SBAC-PAD), pp. 380-387, IEEE, 2018 